\title{
Comparative Antimicrobial Activity of Granulysin against Bacterial Biothreat Agents
}

\author{
Janice J. Endsley ${ }^{\mathrm{a}, \mathrm{c}, *}$, Alfredo G. Torres ${ }^{\mathrm{a}, \mathrm{b}, \mathrm{c}}$, Christine M. Gonzales ${ }^{\mathrm{a}}$, Valeri G. Kosykh ${ }^{\mathrm{b}}$, Vladimir L.

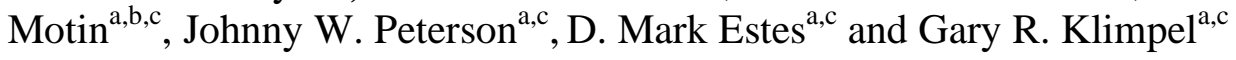

Department of Microbiology and Immunology ${ }^{a}$, Department of Pathology ${ }^{b}$, and Sealy Center for Vaccine Development ${ }^{c}$, University of Texas Medical Branch, Galveston, TX 77555-0436, USA

\begin{abstract}
Granulysin is a cationic protein produced by human $\mathrm{T}$ cells and natural killer cells that can kill bacterial pathogens through disruption of microbial membrane integrity. Herein we demonstrate antimicrobial activity of the granulysin peptide derived from the active site against Bacillus anthracis, Yersinia pestis, Francisella tularensis, and Burkholderia mallei, and show pathogen-specific differences in granulysin peptide effects. The susceptibility of $Y$. pestis to granulysin is temperature dependent, being less susceptible when grown at the flea arthropod vector temperature $\left(26^{\circ} \mathrm{C}\right)$ than when grown at human body temperature. These studies suggest that augmentation of granulysin expression by cytotoxic lymphocytes, or therapeutic application of granulysin peptides, could constitute important strategies for protection against select agent bacterial pathogens. Investigations of the microbial surface molecules that determine susceptibility to granulysin may identify important mechanisms that contribute to pathogenesis.
\end{abstract}

Keywords: Granulysin, antimicrobial, B. anthracis, B. mallei, Y. pestis, F. tularensis.

\section{INTRODUCTION}

Bacteria that evade the host immune response by surviving phagocytosis comprise some of the most serious microbial threats to human health. Among these are Mycobacterium tuberculosis (Mtb) and some of the pathogens classified by the Center for Disease Control and Prevention as bacterial select agents, Bacillus anthracis, Yersinia pestis, Burkholderia mallei, and Francisella tularensis. The tropism for phagocytic cells by these pathogens is an important factor for their successful evasion of the immune system and poses significant challenges for immunization or therapeutic intervention. Characterizing protective immune responses to these pathogens, then, is paramount to development of prophylactic or early therapeutic intervention strategies. Cytotoxic T cells $\left(\mathrm{T}_{\mathrm{c}}\right)$ and NK cells control intracellular pathogens through multiple killing mechanisms, including death receptor activation and release of cytolytic granule proteins. In human cytotoxic lymphocytes, granulysin is an important component of the lytic repertoire and is stored in cytotoxic granules along with perforin and granzymes [1-5] . Perforin and granzymes kill the infected cell, whereas granulysin has the unique capacity to kill the intracellular pathogen by disruption of microbial membranes [5-7]. The lytic activity due to granulysin has been described against several important pathogens, including Mtb, Plasmodium falciparum, Cryptococcus neoformans, Salmonella enterica serovar Typhimurium (S. Typhimurium), Escherichia coli 0157:H7 and Staphylococcus aureus [5-10].

*Address correspondence to this author at the University of Texas Medical Branch, 301 University Blvd., Galveston, TX 77555-0436, USA; Tel: (409) 772-3142; Fax: (409) 747-6869; E-mail: jjendsle@utmb.edu
The ability of granulysin to kill intracellular $M t b$ supports an important role for this molecule in the cytotoxic lymphocyte $\left(\mathrm{T}_{\mathrm{c}}\right.$ and $\mathrm{NK}$ cells) response to intracellular bacterial pathogens that evade the immune response through residence in macrophages. To investigate a potential role for granulysin in the immune repsonse to an expanded list of intracellular bacterial pathogens for which interventions are urgently needed, we utilized a peptide derived from the active site of human granulysin and a negative control peptide. The active site peptide has been previously shown to reproduce the effects of the recombinant granulysin molecule, and is considered to have potential as an antibacterial therapeutic $[5-7,10,11]$. In this study, we demonstrate and compare the antibacterial activity of granulysin against $B$. anthracis (Ames), Y. pestis (CO 92), F. tularensis (SHU S4 and LVS), and B. mallei (ATCC 23344), using a peptide derived from the active site of granulysin. These studies support the need to further characterize the role of granulysin in the innate and acquired CTL response to these important pathogens.

\section{MATERIALS AND METHODS}

Synthetic custom peptides $>95 \%$ pure by HPLC were purchased from New England Peptide LLC (Gardner, MA, USA). A peptide corresponding to amino acid residues 34-55 of human granulysin (CRTGRSRWRDVCRNFMRRYQSR) was synthesized. As a negative control, a peptide corresponding to amino acids 2-22 (RDYRTCLTIVQKLKKMVDKPT) of human granulysin was also synthesized. Lyophilized peptide was stored in desiccant at $-20{ }^{\circ} \mathrm{C}$ prior to use. Peptide stocks $(5 \mathrm{mM})$ were solubilized in $0.1 \mathrm{~N}$ acetic acid solution, and then further diluted in sterile PBS to $1 \mathrm{mM}$, prior to use. Polypropylene tubes were used to store, 
aliquot, or perform experiments with peptides to prevent loss of peptides due to binding to tubes. All experimentation with select agents was performed under level 2 or 3 biosafety conditions according to protocols approved by the University of Texas Medical Branch Environmental Health and Safety. $S$. Typhimurium, F. tularensis, B. anthracis and B. mallei were grown in brain heart infusion broth (BHI), Muller Hinton media with Isovitle X, Luria-Bertani (LB) media, and LB media supplemented with $4 \%$ glucose (LBG), respectively. Cultures of $S$. Typhimurium, $F$. tularensis (LVS, SHU S4), B. anthracis (Ames strain) and B. mallei (ATCC 23344) were grown to exponential phase and diluted in growth medium to approximately $10^{5}$ colony forming units $(\mathrm{CFU}) / \mathrm{ml}$. A $90 \mu \mathrm{l}$ aliquot of diluted microorganism was pre-incubated with $10 \mu \mathrm{l}$ of PBS or $10 \mu \mathrm{l}$ of individual granulysin peptides serially diluted in PBS to final concentrations of 1,10 , and $100 \mu \mathrm{M}$. After 3 hours of incubation at room temperature, $100 \mu \mathrm{l}$ aliquots of $S$. Typhimurium, $F$. tularensis, and B. malle $i$ were plated on LB or LBG agar plates, and $100 \mu \mathrm{l}$ aliquots of peptide and B. anthracis was plated on trypticase soy agar with 5\% sheep blood. CFU's were counted after overnight incubation ( $48 \mathrm{~h}$ for $\mathrm{B}$. mallei) at $37^{\circ} \mathrm{C}$. Exponential growth phase cultures of $Y$. pestis $\mathrm{CO} 92$ or $Y$. pestis CO92 caf were grown overnight at $26^{\circ} \mathrm{C}$ versus $37^{\circ} \mathrm{C}$ in Heart-Infusion Broth (HIB, Difco). Cultures were diluted 1:100 after overnight growth and grown at $26^{\circ} \mathrm{C}$ and $37^{\circ} \mathrm{C}$ to $\log$ phase in HIB medium. Cultures were harvested by centrifugation and washed once with Phosphate buffered saline buffer (PBS, $\mathrm{pH} 7.4)$. Y. pestis $\left(2 \times 10^{6} \mathrm{CFU} /\right.$ $\mathrm{ml}$ ) were incubated with various concentration of granulysin as described above and plated on HIB agar plates. CFU's were counted after $48 \mathrm{~h}$ incubation at $26^{\circ} \mathrm{C}$. Results for all isolates were verified by three (B. mallei, B. anthracis), four (S. Typhimurium, $F$. tularensis), or five ( $Y$. pestis) independent experiments. A total of five independent experiments were performed with $Y$. pestis to firmly establish the temperature-dependent differences in susceptibility to peptide. Data were analyzed by one-way analysis of variance (ANOVA) followed by a Tukey's pair wise comparison test (GraphPad Software v4.0).

\section{RESULTS}

In this study, we tested the antimicrobial activity of the granulysin peptide against four aerosol-acquired select agent bacterial pathogens, B. anthracis, $Y$. pestis, F. tularensis, and B. mallei. S. Typhimurium was used as a positive control to compare the relative antibacterial activity of the peptide observed in earlier studies $[7,10,12]$. To normalize data across experiments and organisms, results in Fig. (1) are shown as percentage of control growth from each individual experiment. Statistically significant differences due to treatment, however, were determined using actual CFU relative to peptide concentrations. A peptide derived from a region in granulysin previously determined to lack antimicrobial activity (helix 1) was used as a negative control $[6,7,10]$.

In support of previous studies with peptide and recombinant protein $[7,10,12]$, we observed that granulysin peptide had potent antimicrobial activity against $S$. Typhimurium with a significant, dose dependent, increase in activity from 1 to $100 \mu \mathrm{M}$ peptide concentration (Fig. 1). A significant reduction of $B$. anthracis, $F$. tularensis, and $B$. mallei was evident at 10 and $100 \mu \mathrm{M}$ of peptide (Fig. 1), though $B$. anthracis and $F$. tularensis were less susceptible to 100 $\mu \mathrm{M}$ levels than $B$. mallei or $S$. Typhimurium. The concentration of peptide required to reduce $\mathrm{CFU}$ of $B$. anthracis and $F$. tularensis was comparable to peptide concentrations required to reduce $M t b$ and $M$. bovis, in similar studies [5, 7, 12]. The killing activity of granulysin against $F$. tularensis SHU S4 (virulent) and LVS (attenuated vaccine strain) were similar (data not shown) indicating that susceptibility to granulysin peptide is not a likely variable in pathogenesis. Surprisingly, the negative control peptide derived from helix 1 had moderate activity against $B$. anthracis at a concentration of $100 \mu \mathrm{M}$ (38\% reduction of CFU's as compared to the no peptide control; data not shown). The growth of $S$. Typhimurium, $Y$. pestis, F. tularensis, or B. mallei, was not affected by the helix 1 peptide when compared to growth in the absence of peptide. Due to the effects of the helix 1 peptide on B. anthracis, the no peptide control CFUs were used to calculate the percentage of control growth shown in Fig. (1). As shown in Fig. (2), Y. pestis growth was also

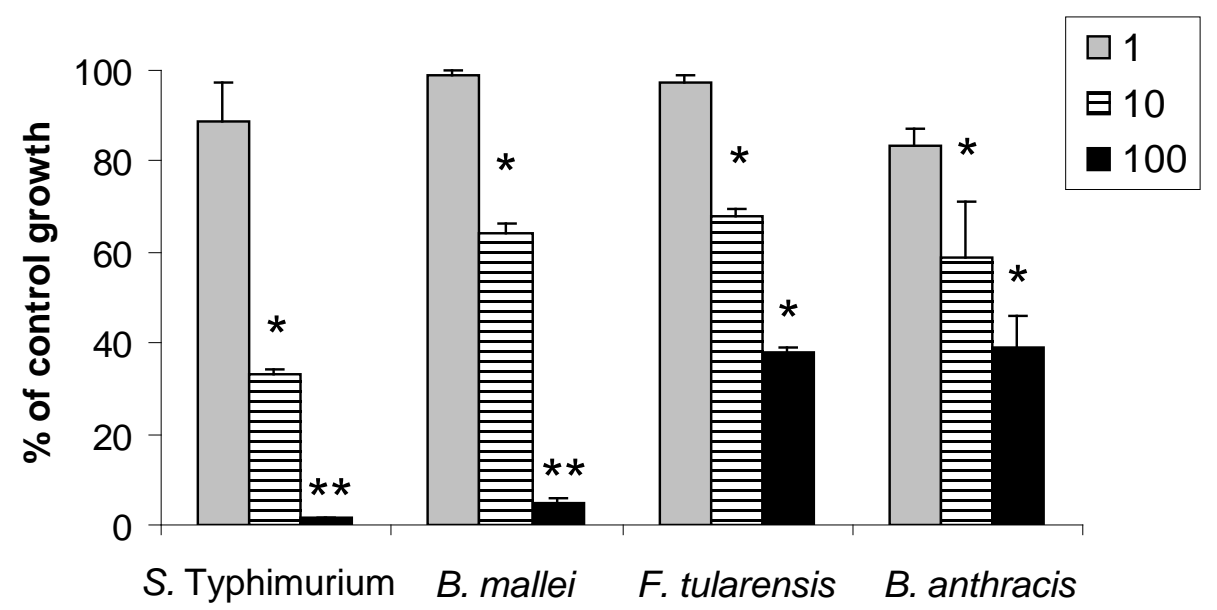

Fig. (1). Antimicrobial activity of granulysin peptide against select agent pathogens. Reduction of $S$. Typhimurium, B. mallei, F. tularensis (SHU S4), and B. anthracis (Ames) by a peptide derived from the active site of granulysin $(1,10,100 \mu \mathrm{M})$ displayed as a percentage of control (no peptide) growth. Data shown are mean \pm SEM of three to four independent experiments performed in triplicate. $* \mathrm{p}<0.05 ; * * \mathrm{p}<0.01$, indicate statistically significant differences between peptide treatment and negative control. 


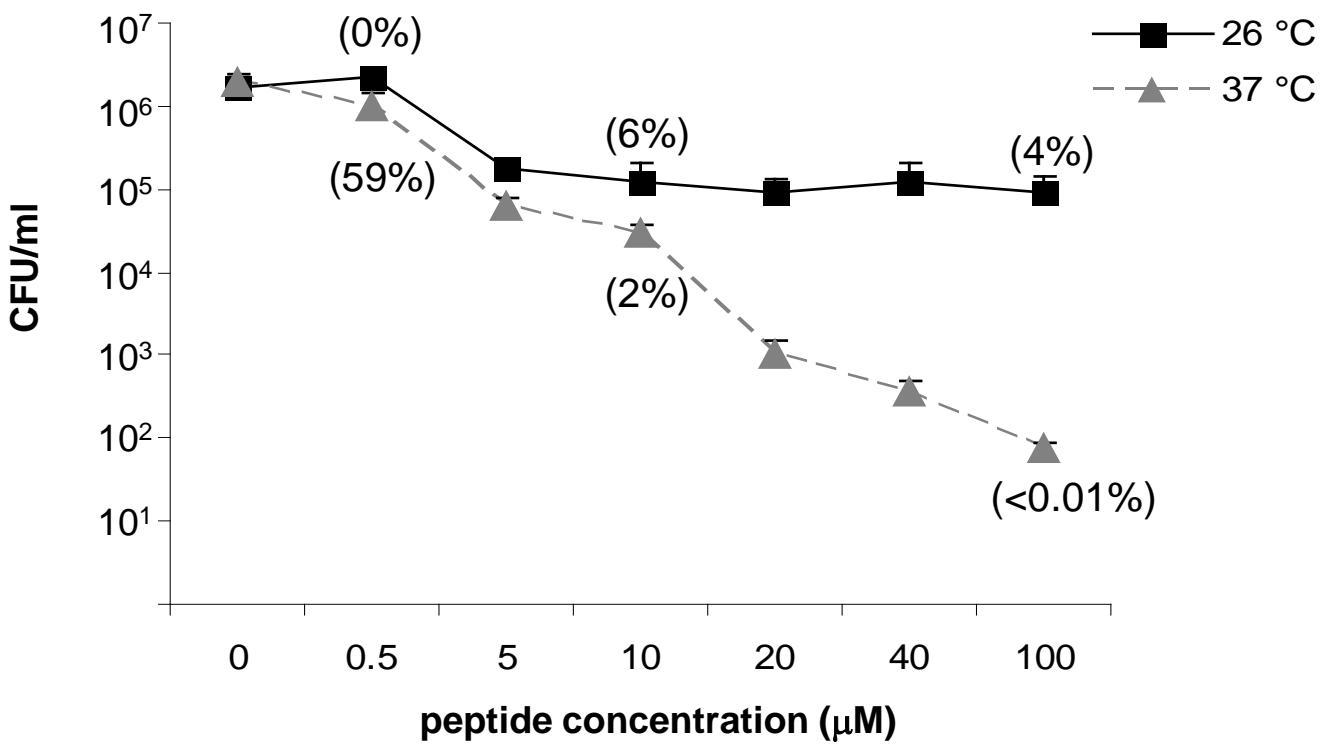

Fig. (2). Activity of granulysin peptide against $Y$. pestis is growth temperature-dependent. CFU reduction of $Y$. pestis (CO 92) across granulysin peptide concentration when grown at $26^{\circ} \mathrm{C}$ and $37^{\circ} \mathrm{C}$. Reduction of CFU following $3 \mathrm{~h}$ incubation with peptide was determined by overnight growth on HIB agar plates. Percentage reduction from control growth at representative peptide concentrations is shown in parenthesis. Data shown are mean \pm SEM of five independent experiments. Compared to negative control, growth was significantly ( $<<0.01$ ) reduced by 5 to $100 \mu \mathrm{M}$ concentration of granulysin peptide at both 26 and $37^{\circ} \mathrm{C}$. Effects of peptide on growth reduction were significantly different $(\mathrm{p}<0.05)$ at $26^{\circ} \mathrm{C}$ compared to $37^{\circ} \mathrm{C}$ from 5 to $100 \mu \mathrm{M}$ concentration.

significantly reduced by the granulysin active site peptide in a dose-dependent manner. A growth temperature-dependent susceptibility of $Y$. pestis to cationic peptides has previously been characterized [13-15]. To determine if this effect could be observed with granulysin, $Y$. pestis was incubated with the peptide after growth at ambient temperature, generally corresponding to that in the flea arthropod vector $\left(26^{\circ} \mathrm{C}\right)$, or human body temperature $\left(37^{\circ} \mathrm{C}\right)$. When grown at ambient temperature to exponential growth phase, $Y$. pestis was much more resistant to the effects of granulysin peptide. In fact, the reduction of $Y$. pestis growth by granulysin peptide was several orders of magnitude greater when grown at $37^{\circ} \mathrm{C}$, as compared to growth at $26^{\circ} \mathrm{C}$ (Fig. 2). As a percentage of the negative control, however, significant reduction of $Y$. pestis by 5 to $100 \mu \mathrm{M}$ concentrations of granulysin peptide was evident at both temperatures (Fig. 2, numbers in parenthesis). Recently, surface-exposed bacterial molecules (capsular antigen fraction 1) have been shown to modulate the susceptibility of $Y$. pestis to antimicrobial molecules in the respiratory epithelium [16]. To evaluate the role of the capsule substance in the susceptibility of $Y$. pestis to granulysin, a capsule-negative mutant of $Y$. pestis $\mathrm{CO} 92$ (Y. pestis CO 92 caf) was also grown at both temperatures prior to incubation with peptide. The mutant contains a 1,176 bp deletion in the caf-operon eliminating synthesis of Caf1 capsular subunit and Caf1A usher proteins (V. Motin, unpublished). A temperature-dependent difference in killing activity by $Y$. pestis CO 92 caf was observed similar to the wild-type strain shown in Fig. (2), while the presence or absence of the capsule did not affect the susceptibility (data not shown). These differences in susceptibility to the antimicrobial effects of granulysin peptide, due to temperature, were not caused by altered growth patterns as organisms grew to similar density at both temperatures (Fig. 2).

\section{DISCUSSION}

The identification of immune mechanisms that can protect against bacterial select agent pathogens is a critical component in the development of control measures for these potential biothreats. The important role of both innate and acquired cell-mediated immune (CMI) responses to intracellular pathogens strongly supports the need to characterize CMI mechanisms that can be targeted to prevent disease or improve clinical outcome. In this study we showed that granulysin, a cytotoxic lymphocyte-derived antimicrobial, may have an important role in immunity to several important intracellular bacteria with select agent status.

Granulysin is a cationic molecule that consists of five compact $\alpha$-helical segments. Homologues of granulysin in multiple mammalian species, fish, and birds indicates conservation of this antimicrobial mechanism [2, 12, 17-21]. Curiously, a granulysin homologue has not been identified in mice, hampering studies of the effects of gene deletion. In contrast to other antimicrobial molecules of the immune system, granulysin expression is limited to NK cells and antigen-specific $\mathrm{T}$ cells, the cytotoxic component of the CMI response. Expression is constitutive in NK cells and inducible in antigen specific $\mathrm{T}$ cells following activation with specific antigen or cytokines[1-3]. Native granulysin has chemoattractant capabilities [22] and has recently been implicated in adverse drug reactions that affect epidermal cells [23]. Normally, granulysin does not have cytotoxic effects on non-transformed human cells, while induction of apoptosis of tumor cells has been shown by several groups $[10,24,25]$. Peptide mapping in three separate species has characterized a core amino acid region including residues in helix 2 through helix 3 as the lytic site $[6,7,10,12]$. The localization of the antimicrobial activity of granulysin to a short amino acid segment indicates the potential for use as a 
therapuetic peptide, as has been described for other small "killer" peptides [26]. Peptides derived from the lytic site of granulysin are able to reproduce the antimicrobial effects of recombinant granulysin. The activity of the derived peptide can exceed the recombinant molecule $[5,7]$, an effect that may be due to the harsh denaturing conditions required to purify the recombinant molecule or to a membrane contact advantage of the peptide. Nonetheless, this peptide is an important screening tool to identify the antibacterial potential of granulysin against different bacterial pathogens and may also have utility as a bactericidal therapeutic. Granulysinderived peptides have been shown to neutralize LPS simultaneously with direct bacterial killing and may have potential as a treatment for septic shock [11]. In the current study we also observed antimicrobial effects of the peptide derived from helix 1 of granulysin against $B$. anthracis. This result was unexpected, as peptides corresponding to the helix 1 region of granulysin have previously been reported to have no antimicrobial activity [6, 7, 10] Following elucidation of the crystal structure of granulysin, however, the helix 1 region was predicted to contribute to the antimicrobial activity of granulysin due to the distribution and orientation of positively charged amino acids in this region [27]. Further studies with peptides and site specific amino acid substitution in recombinant protein are needed to fully characterize the lytic sites utilized by granulysin against various classes of microbial pathogens.

Our results demonstrate that granulysin may have very potent lytic effects on $Y$. pestis and $B$. mallei and can reduce the growth of $F$. tularensis and B. anthracis at higher concentrations. The relative potency of granulysin to reduce bacterial numbers in the current study varied by organism, and in regards to $Y$. pestis, the effect is temperature dependent. Collectively, the results from these and other studies suggest that the surface-exposed structures on microbial membranes that determine susceptibility to granulysin could differ among microorganisms, or vary in response to environmental signals. Peptides derived from granulysin are able to bind LPS and neutralize LPS-induced secretion of TNF $\alpha$ by peripheral blood mononuclear cells [11]. Differences in acylation of the LPS lipid A moiety due to environmental temperature is a defined mechanism for bacterial resistance to antimicrobial peptides [28]. Antimicrobial peptides have a significant role in insect host defense and are an important selective pressure for survival of bacteria that utilize insect reservoirs. With regard to $Y$. pestis, growth at environmental temperature increases the resistance of $Y$. pestis to cationic molecules with antimicrobial activity [13-15]. These differences are frequently attributed to temperatureinduced alterations in lipid A acylation [13-15, 29], though acyl transferase deficiency has no effect on $Y$. pestis susceptibility to polymyxin B [30]. Very recently, bacterial capsule polysaccharides from several species were demonstrated to mediate resistance to both polymyxin B and alpha-defensin from human neutrophils and were proposed to act as bacterial decoys for antibacterial peptides [31]. Susceptibility of $Y$. pestis to beta-defensin and cathelicidin was also recently shown to be mediated by the capsular antigen fraction [16]. In our studies, a $Y$. pestis variant deficient for the capsule substance was not more susceptible to granulysin peptide effects. Thus, the role of capsule polysaccharides as bacterial decoys may be an important difference between granulysin and other cationic antimicrobial molecules.

As previously observed, high concentrations of granulysin are required to kill micro-organisms in bulk culture compared to antibiotics or many synthetic antimicrobials [5, $7,12]$. An important difference between granulysin and other antimicrobials is that NK cells and antigen-specific T cells deliver granulysin to individual target cells at sites of infection. These cytotoxic immune cells are able to methodically kill one infected target at a time using only a portion of available granules while continually replenishing the granule armament. Perforin functions to increase membrane permeability of target cells to facilitate entry and activity of granulysin and granzymes [5, 32, 33]. Granulysin molecules are able to directly disrupt microbial membranes by electrostatic charge disruption [7, 27, 34]. Granulysin can also elicit extra-cellular microbicidal effects against pathogens $[5,9]$. In this regard, both virulent Francisella and LVS were shown to have a significant extra-cellular phase in their in vivo life cycle [35].

The antibacterial effects of the granulysin peptide observed in the current study support continued investigation of granulysin as an important cytotoxic effector molecule contributing to the protective CMI responses to these and other serious biothreats. Promoting activation of granulysin expression by NK cells and T cells could represent an important avenue for immune modulation to protect susceptible populations from weaponized or naturally occurring bacterial pathogens. Strategies to promote activation of NK cell antimicrobial activity may be very effective in the early innate immune response to several pathogens. Promoting antimicrobial protein expression as part of the effector repertoire of antigen-specific $\mathrm{T}$ cells may also improve vaccine efficacy. In summary, induction of granulysin by NK cells or antigenspecific $\mathrm{T}$ cells should be further characterized as a mechanism to augment protective immune function against intracellular bacterial pathogens.

\section{ACKNOWLEDGEMENTS}

This study was supported by the Department of Microbiology and Immunology and The Sealy Center for Vaccine Development, The University of Texas Medical Branch.

\section{REFERENCES}

[1] Houchins JP, Kricek F, Chujor CS, et al. Genomic structure of NKG5, a human NK and T cell-specific activation gene. Immunogenetics 1993; 37: 102-7.

[2] Jongstra J, Schall TJ, Dyer BJ, et al. The isolation and sequence of a novel gene from a human functional T cell line. J Exp Med 1987; 165: 601-14.

[3] Yabe T, McSherry C, Bach FH, Houchins JP. A cDNA clone expressed in natural killer and $\mathrm{T}$ cells that likely encodes a secreted protein. J Exp Med 1990; 172: 1159-63.

[4] Stegelmann F, Bastian M, Swoboda K, et al. Coordinate expression of $\mathrm{CC}$ chemokine ligand 5, granulysin, and perforin in $\mathrm{CD}^{+} \mathrm{T}$ cells provides a host defense mechanism against Mycobacterium tuberculosis. J Immunol 2005; 175: 7474-83.

[5] Stenger S, Hanson DA, Teitelbaum R, et al. An antimicrobial activity of cytolytic T cells mediated by granulysin. Science 1998; 282: $121-5$.

[6] Andreu D, Carreno C, Linde C, Boman HG, Andersson M. Identification of an anti-mycobacterial domain in NK-lysin and granulysin. Biochem J 1999; 344 (Pt 3): 845-9. 
[7] Ernst WA, Thoma-Uszynski S, Teitelbaum R, et al. Granulysin, a $\mathrm{T}$ cell product, kills bacteria by altering membrane permeability. $\mathrm{J}$ Immunol 2000; 165: 7102-8.

[8] Farouk SE, Mincheva-Nilsson L, Krensky AM, Dieli F, TroyeBlomberg M. Gamma delta $\mathrm{T}$ cells inhibit in vitro growth of the asexual blood stages of Plasmodium falciparum by a granule exocytosis-dependent cytotoxic pathway that requires granulysin. Eur J Immunol 2004; 34: 2248-56.

[9] Ma LL, Spurrell JC, Wang JF, et al. CD8 T cell-mediated killing of Cryptococcus neoformans requires granulysin and is dependent on CD4 T cells and IL-15. J Immunol 2002; 169: 5787-95.

[10] Wang Z, Choice E, Kaspar A, et al. Bactericidal and tumoricidal activities of synthetic peptides derived from granulysin. J Immunol 2000; 165: 1486-90.

[11] Chen X, Howe J, Andra J, et al. Biophysical analysis of the interaction of granulysin-derived peptides with enterobacterial endotoxins. Biochim Biophys Acta 2007; 1768: 2421-31.

[12] Endsley JJ, Furrer JL, Endsley MA, et al. Characterization of bovine homologues of granulysin and NK-lysin. J Immunol 2004; 173: 2607-14.

[13] Bengoechea JA, Lindner B, Seydel U, Diaz R, Moriyon I. Yersinia pseudotuberculosis and Yersinia pestis are more resistant to bactericidal cationic peptides than Yersinia enterocolitica. Microbiology 1998; 144: 1509-15.

[14] Anisimov AP, Dentovskaya SV, Titareva GM, et al. Intraspecies and temperature-dependent variations in susceptibility of Yersinia pestis to the bactericidal action of serum and to polymyxin B. Infect Immun 2005; 73: 7324-31.

[15] Bengoechea JA, Diaz R, Moriyon I. Outer membrane differences between pathogenic and environmental Yersinia enterocolitica biogroups probed with hydrophobic permeants and polycationic peptides. Infect Immun 1996; 64: 4891-9.

[16] Galvan EM, Lasaro MA, Schifferli DM. Capsular antigen fraction 1 and Pla modulate the susceptibility of Yersinia pestis to pulmonary antimicrobial peptides such as cathelicidin. Infect Immun 2008; 76: 1456-64.

[17] Andersson M, Gunne H, Agerberth B, et al. NK-lysin, a novel effector peptide of cytotoxic T and NK cells. Structure and cDNA cloning of the porcine form, induction by interleukin 2, antibacterial and antitumour activity. EMBO J 1995; 14: 1615-25.

[18] Davis EG, Sang Y, Rush B, Zhang G, Blecha F. Molecular cloning and characterization of equine NK-lysin. Vet Immunol Immunopathol 2005; 105: 163-9.

[19] Hong YH, Lillehoj HS, Dalloul RA, et al. Molecular cloning and characterization of chicken NK-lysin. Vet Immunol Immunopathol 2006; 110: 339-47.

[20] Wang Q, Wang Y, Xu P, Liu Z. NK-lysin of channel catfish: gene triplication, sequence variation, and expression analysis. Mol Immunol 2006; 43: 1676-86.
[21] Praveen K, Evans DL, Jaso-Friedmann L. Evidence for the existence of granzyme-like serine proteases in teleost cytotoxic cells. J Mol Evol 2004; 58: 449-59.

[22] Deng A, Chen S, Li Q, Lyu SC, Clayberger C, Krensky AM. Granulysin, a cytolytic molecule, is also a chemoattractant and proinflammatory activator. J Immunol 2005; 174: 5243-8.

[23] Chung WH, Hung SI, Yang JY, et al. Granulysin is a key mediator for disseminated keratinocyte death in Stevens-Johnson syndrome and toxic epidermal necrolysis. Nat Med 2008; 14: 1343-50.

[24] Gamen S, Hanson DA, Kaspar A, Naval J, Krensky AM, Anel A. Granulysin-induced apoptosis. I. Involvement of at least two distinct pathways. J Immunol 1998; 161: 1758-64

[25] Okada S, Li Q, Whitin JC, Clayberger C, Krensky AM. Intracellular mediators of granulysin-induced cell death. J Immunol 2003; 171: 2556-62.

[26] Savoia D, Scutera S, Raimondo S, Conti S, Magliani W, Polonelli L. Activity of an engineered synthetic killer peptide on Leishmania major and Leishmania infantum promastigotes. Exp Parasitol 2006; 113: 186-92.

[27] Anderson DH, Sawaya MR, Cascio D, et al. Granulysin crystal structure and a structure-derived lytic mechanism. J Mol Biol 2003; 325: 355-65.

[28] Guo L, Lim KB, Poduje CM, et al. Lipid A acylation and bacterial resistance against vertebrate antimicrobial peptides. Cell 1998; 95 : 189-98.

[29] Bengoechea JA, Brandenburg K, Arraiza MD, Seydel U, Skurnik M, Moriyón I. Pathogenic Yersinia enterocolitica strains increase the outer membrane permeability in response to environmental stimuli by modulating lipopolysaccharide fluidity and lipid A structure. Infect Immun 2003; 71: 2014-21.

[30] Rebeil R, Ernst RK, Jarrett CO, Adams KN, Miller SI, Hinnebusch BJ. Characterization of late acyltransferase genes of Yersinia pestis and their role in temperature-dependent lipid A variation. J Bacteriol 2006; 188: 1381-8

[31] Llobet E, Tomas JM, Bengoechea JA. Capsule polysaccharide is a bacterial decoy for antimicrobial peptides. Microbiology 2008; 154: $3877-86$

[32] Lieberman J. The ABCs of granule-mediated cytotoxicity: new weapons in the arsenal. Nat Rev Immunol 2003; 3: 361-70.

[33] Walch M, Latinovic-Golic S, Velic A, et al. Perforin enhances the granulysin-induced lysis of Listeria innocua in human dendritic cells. BMC Immunol 2007; 8: 14.

[34] Miteva M, Andersson M, Karshikoff A, Otting G. Molecular electroporation: a unifying concept for the description of membrane pore formation by antibacterial peptides, exemplified with NKlysin. FEBS Lett 1999; 462: 155-8.

[35] Forestal CA, Malik M, Catlett SV, et al. Francisella tularensis has a significant extracellular phase in infected mice. J Infect Dis 2007; 196: $134-7$. 